\title{
Fundamental studies of relationships between experimental nonlinear coherent vibrational spectroscopies \\ Supporting Information
}

\author{
John C. Wright \\ Department of Chemistry \\ University of Wisconsin-Madison \\ 1101 University Avenue \\ Madison, WI 53706
}

Derivation of temporal coherence evolution after step function excitation

The Liouville equation defines the evolution of an initial coherence, $\rho_{k j}$ or $\rho_{i k}$, to a subsequent coherence, $\rho_{i j}$.

$\dot{\rho}_{i j}=-i \delta_{i j} \rho_{i j}+\frac{i}{2}\left(e^{i(\vec{k} \cdot \vec{z}-\omega t)}+e^{-i(\vec{k} \cdot \vec{z}-\omega t)}\right)\left(\Omega_{i k} \rho_{k j}-\Omega_{j k} \rho_{i k}\right)$.

The frequency of the feeding coherences, $\rho_{k j}$ or $\rho_{i k}$, depends upon whether they are driven by an excitation field, $\omega_{1}$, or defined by free induction decay, $\omega_{k j}$ or $\omega_{i k}$. We then assume that $\rho_{\mathrm{kj}}$ and $\rho_{\mathrm{ik}}$ have the form

$$
\rho_{k j}=\tilde{\rho}_{k j} e^{-i \delta_{k j} t} \text { and } \rho_{i k}=\tilde{\rho}_{i k} e^{-i \delta_{i k} t}
$$

where $\tilde{\rho}_{i k}$ or $\tilde{\rho}_{k j}$ contain the $e^{i \vec{k} \cdot \vec{z}}$ dependence and $\delta_{m n} \equiv \pm \omega_{1}-i \Gamma_{m n}$ for the driven coherence or $\delta_{m n} \equiv \omega_{m n}-i \Gamma_{m n}$ for the free induction decay. The sign of $\omega_{1}$ depends on the sign of $\omega_{m n}$. Substituting (2) into (1), we obtain

$\dot{\rho}_{i j}=-i \delta_{i j} \rho_{i j}+\frac{i}{2}\left(e^{i(\vec{k} \cdot \vec{z} \cdot \omega t)}+e^{-i(\vec{k} \cdot \vec{z}-\omega t)}\right)\left(\Omega_{i k} \tilde{\rho}_{k j} e^{-i \delta_{k_{j}} t}-\Omega_{j k} \tilde{\rho}_{i k} e^{-i \delta_{i k} t}\right)$

Expanding this expression and combining the exponents,

$\dot{\rho}_{i j}=-i \delta_{i j} \rho_{i j}+\frac{i}{2}\left[\begin{array}{l}\Omega_{i k} \tilde{\rho}_{k j}\left(e^{i\left[\vec{k} \cdot \vec{z}-\left(\delta_{i j}+\omega\right) t\right]}+e^{-i\left[\vec{k} \cdot \vec{z}-\left(\delta_{k j}+\omega\right) t\right]} e^{-i\left(\delta_{j j}-\omega\right) t}\right) \\ -\Omega_{j k} \tilde{\rho}_{i k}\left(e^{i\left[\vec{k} \cdot \bar{z}-\left(\delta_{i k}+\omega\right) t\right]}+e^{-i\left[\vec{k} \cdot \bar{z}-\left(\delta_{i k}+\omega\right) t\right]} e^{-i\left(\delta_{k j}-\omega\right) t}\right)\end{array}\right]$

We assume the Rabi frequency results from a step function excitation pulse and solve the differential equation using Laplace transforms. 


$$
\left(s+i \delta_{i j}\right) \rho_{i j}-e^{i\left[\left( \pm \vec{k}_{1}+\vec{k}_{2}\right) \cdot \vec{z}\right]} \rho_{i j}^{o}=\frac{i e^{i \vec{k}_{2} \cdot \vec{z}}}{2}\left(\Omega_{i k} \tilde{\rho}_{k j}\left(\frac{1}{s+i\left(\delta_{k j}+\omega\right)}+\frac{1}{s+i\left(\delta_{k j}-\omega\right)}\right)\right)
$$

Note that it was necessary to explicitly include the spatial dependence of the phase in the FID component of the initial value of the final $\rho_{i j}$ coherence which depends on the kvectors of the initial and final coherences. Solving for the coherence,

$$
\rho_{i j}=\frac{e^{i\left[\left( \pm \vec{k}_{1}+\vec{k}_{2}\right) \cdot \vec{z}\right]} \rho_{i j}^{o}}{\left(s+i \delta_{i j}\right)}+\frac{i}{2}\left(\begin{array}{l}
\Omega_{i k} \tilde{\rho}_{k j}\left(\frac{1}{\left[s+i\left(\delta_{k j}+\omega\right)\right]\left[s+i \delta_{i j}\right]}+\frac{1}{\left[s+i\left(\delta_{k j}-\omega\right)\right]\left[s+i \delta_{i j}\right]}\right) \\
\left.-\Omega_{j k} \tilde{\rho}_{i k}\left(\frac{1}{\left[s+i\left(\delta_{i k}+\omega\right)\right]\left[s+i \delta_{i j}\right]}+\frac{1}{\left[s+i\left(\delta_{i k}-\omega\right)\right]\left[s+i \delta_{i j}\right]}\right)\right)
\end{array}\right)
$$

Taking the inverse Laplace transform,

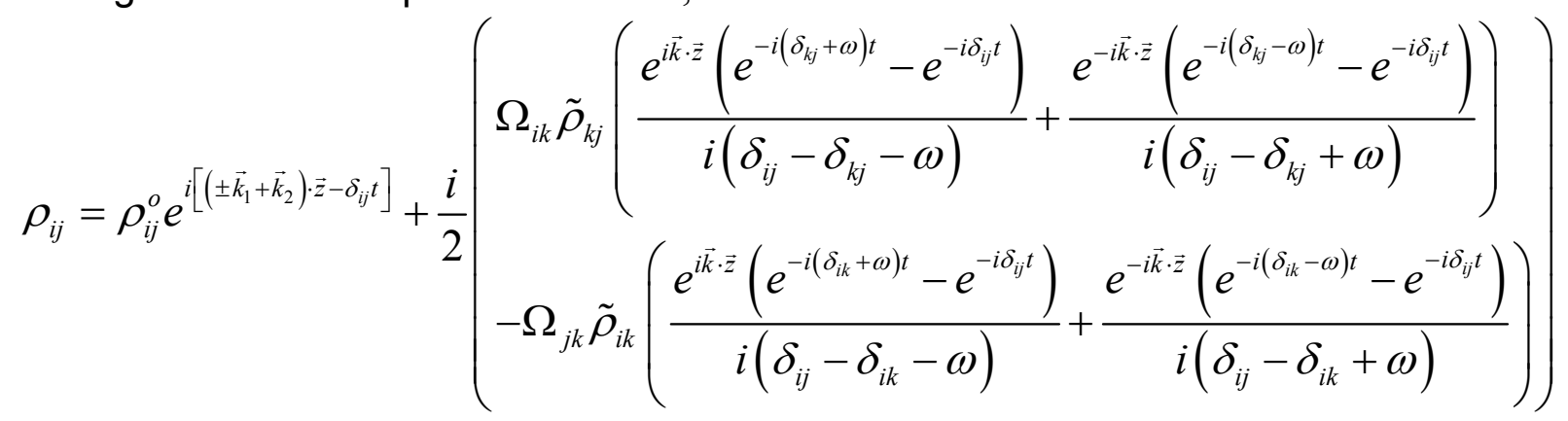

For FID of the initial coherence,

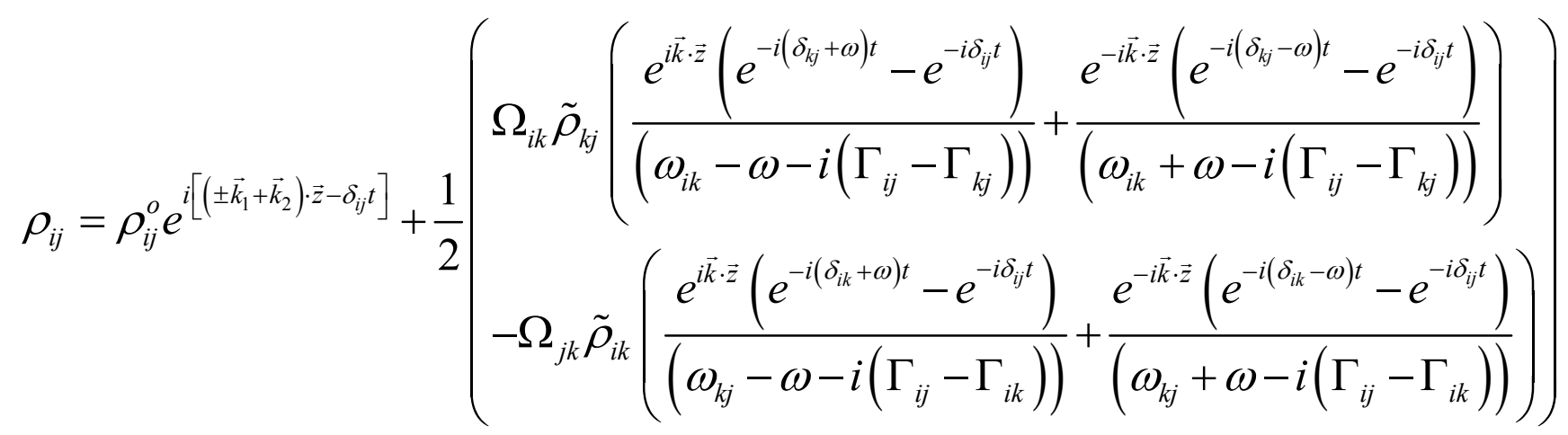

(8)

For an initial coherence driven at $\omega_{1}$, 


$$
\rho_{i j}=\rho_{i j}^{o} e^{-i \delta_{i j} t}+\frac{i}{2}\left(\begin{array}{l}
\Omega_{i k} \tilde{\rho}_{k j}\left(\frac{e^{i \vec{k}_{2} \cdot \vec{z}}\left(e^{-i\left( \pm \omega_{1}+\omega-i \Gamma_{k j}\right) t}-e^{-i \delta_{i j} t}\right)}{i\left(\omega_{i j} \mp \omega_{1}-\omega_{2}-i\left(\Gamma_{i j}-\Gamma_{k j}\right)\right)}+\frac{e^{-i \vec{k}_{2} \cdot \vec{z}}\left(e^{-i\left( \pm \omega_{1}-\omega-i \Gamma_{k j}\right) t}-e^{-i \delta_{i j} t}\right)}{i\left(\omega_{i j} \mp \omega_{1}+\omega_{2}-i\left(\Gamma_{i j}-\Gamma_{k j}\right)\right)}\right) \\
-\Omega_{j k} \tilde{\rho}_{i k}\left(\frac{e^{i \vec{k}_{2} \cdot \vec{z}}\left(e^{-i\left( \pm \omega_{1}+\omega-i \Gamma_{i k}\right) t}-e^{-i \delta_{i j} t}\right)}{i\left(\omega_{i j} \mp \omega_{1}-\omega_{2}-i\left(\Gamma_{i j}-\Gamma_{k j}\right)\right)}+\frac{e^{-i \vec{k}_{2} \cdot \vec{z}}\left(e^{-i\left( \pm \omega_{1}-\omega-i \Gamma_{i k}\right) t}-e^{-i \delta_{i j} t}\right)}{i\left(\omega_{i j} \mp \omega_{1}+\omega_{2}-i\left(\Gamma_{i j}-\Gamma_{k j}\right)\right)}\right)
\end{array}\right) .
$$

Note that the $k$-vectors of the initial coherence (defined by $\tilde{\rho}$ ) and the excitation field will add or subtract and define the phase of the resulting coherence. At resonance,

$$
\rho_{i j}=\rho_{i j}^{o} e^{i\left[\left( \pm \vec{k}_{1}+\vec{k}_{2}\right) \cdot \vec{z}-\delta_{i j} t\right]}-\frac{e^{i\left(\vec{k}_{2} \cdot \vec{z}-\omega_{i j} t\right)}}{2}\left(\begin{array}{l}
\Omega_{i k} \tilde{\rho}_{k j}\left(\frac{e^{-\Gamma_{k j} t}-e^{-\Gamma_{i j} t}}{i\left(\Gamma_{i j}-\Gamma_{i k}\right)}+\frac{e^{-\Gamma_{k j} t}-e^{-\Gamma_{i j} t}}{i\left(\Gamma_{i j}-\Gamma_{i k}\right)}\right) \\
-\Omega_{j k} \tilde{\rho}_{i k}\left(\frac{e^{-\Gamma_{i k} t}-e^{-\Gamma_{i j} t}}{i\left(\Gamma_{i j}-\Gamma_{i k}\right)}+\frac{e^{-\Gamma_{i k} t}-e^{-\Gamma_{i j} t}}{i\left(\Gamma_{i j}-\Gamma_{i k}\right)}\right)
\end{array}\right) .
$$

Let's now understand these expressions. For simplicity, we assume the $\tilde{\rho}_{k j}$ or $\tilde{\rho}_{i k}$ coherences do not decay. Then, the first term is the exponential decay of the initial coherence $\rho_{i j}^{o}$ in the absence of any driving field. It has an oscillatory component and a decay component:

$$
\rho_{i j}=\rho_{i j}^{o} e^{-i \omega_{i j} t} e^{-\Gamma_{i j} t} \text {. }
$$

The next four terms correspond to the 4 different cases that are possible when either the ket- or bra-side state undergoes either an absorption or emission. Each case has two terms. The first term corresponds to the steady state response and the second corresponds to the transient response that dies away exponentially because it has the same form as equation (9). An example of the temporal response for the real part of the coherence is shown below. The imaginary part is identical except for a $90^{\circ}$ phase shift. The coherence builds up to a steady state value.

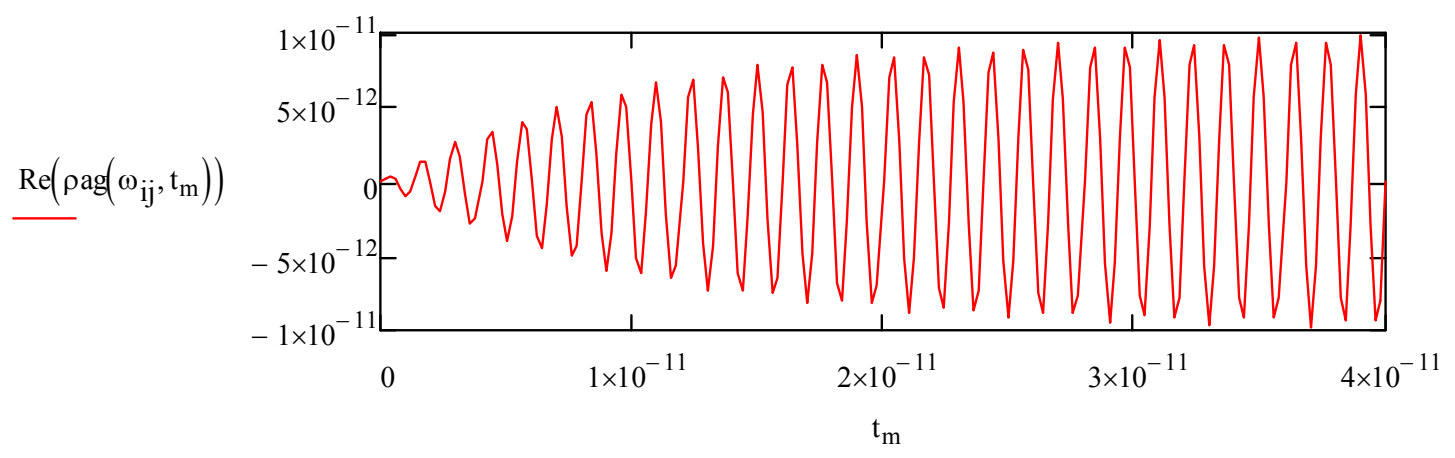

The interpretation of the 4 cases depends on the resonance condition defined by the excitation frequency required to minimize the denominators. At resonance, the 
coherence reaches its maximum. The first two cases correspond to ket-side transitions and the last two cases correspond to bra-side transitions. They have opposite signs. In our diagrams that show the transitions between states, we will use solid arrows for ketside transitions and dotted arrows for bra-side transitions. The diagrams for each case are show below. Again, remember that for each case, the frequencies of the initial coherence ( $\rho_{\mathrm{kj}}$ or $\rho_{\mathrm{ik}}$ ) can be either the free induction decay frequency and/or the driven frequency and we neglect decay of the initial coherence.
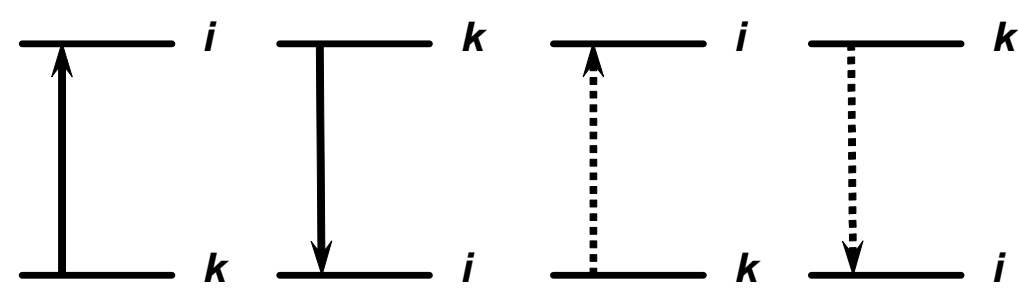

\section{Case 1 Case 2 Case $3 \quad$ Case 4}

Case 1- ket-side absorption transition from state $k$ to state $i$.

This case corresponds to the term

$\rho_{i j}=\frac{\Omega_{k i} \tilde{\rho}_{k j}\left(e^{-i\left(\omega_{k j}+\omega\right) t}-e^{-i \delta_{j i} t}\right)}{2\left(\delta_{i j}-\omega-\omega_{k j}\right)}$

It reaches its minimum value of $-i \Gamma_{i j}$ when $\omega=\omega_{i j}-\omega_{k j}=\omega_{i k}$. For this condition to be met, state $i$ must have a higher energy than state $k$ so the $k \rightarrow i$ transition must be an absorption. That also means that the second term in equation (8) cannot reach resonance because $\omega$ has the opposite sign. The term is antiresonant and corresponds to the term that is ignored in the rotating wave approximation.

Case 2- ket-side emissive transition from state $k$ to state $i$.

This case corresponds to the term

$\rho_{i j}=\frac{\Omega_{i k} \tilde{\rho}_{k j}\left(e^{-i\left(\omega_{k j}-\omega\right) t}-e^{-i \delta_{i j} t}\right)}{2\left(\delta_{i j}+\omega-\omega_{k j}\right)}$

It reaches its minimum value of $-i \Gamma_{i j}$ when $\omega=\omega_{k j}-\omega_{i j}=\omega_{k i}$. For this condition to be met, state $i$ must have a lower energy than state $k$ so the $k \rightarrow i$ transition must be an emission. That also means that the first term in equation (8) cannot reach resonance because has the opposite sign. The term is antiresonant and corresponds to the term that is ignored in the rotating wave approximation.

Case 3- bra-side emission transition from state $k$ to state $j$.

This case corresponds to the term 
$\rho_{i j}=-\frac{\Omega_{j k} \tilde{\rho}_{i k}\left(e^{-i\left(\omega_{i k}+\omega\right) t}-e^{-i \delta_{i j} t}\right)}{2\left(\delta_{i j}-\omega-\omega_{i k}\right)}$

It reaches its minimum value of $-i \Gamma_{i j}$ when $\omega=\omega_{i j}-\omega_{i k}=\omega_{k j}$. For this condition to be met, state $j$ must have a lower energy than state $k$ so the transition must be an emission. That also means that the fourth term in equation (8) cannot reach resonance because $\omega$ has the opposite sign. The term is antiresonant and corresponds to the term that is ignored in the rotating wave approximation.

Case 4- bra-side absorption transition from state $k$ to state $j$.

This case corresponds to the term

$\rho_{i j}=-\frac{\Omega_{j k} \tilde{\rho}_{i k}\left(e^{-i\left(\omega_{i k}-\omega\right) t}-e^{-i \delta_{i j} t}\right)}{2\left(\delta_{i j}+\omega-\omega_{i k}\right)}$

It reaches its minimum value of $-i \Gamma_{i j}$ when $\omega=\omega_{i k}-\omega_{i j}=\omega_{j k}$. For this condition to be met, state $j$ must have a higher energy than state $k$ so the transition must be an absorption. That also means that the third term in equation (8) cannot reach resonance because $\omega$ has the opposite sign. The term is antiresonant and corresponds to the term that is ignored in the rotating wave approximation.

Temporal coherence evolution after square excitation pulse

A square excitation pulse with a width of $\tau$ can be represented by the equation (8) when $t=\tau$ and the response given by (9) where

$\rho_{i j}=\rho_{i j}(t=\tau) e^{-i \omega_{i j}(t-\tau)} e^{-\Gamma_{i j}(t-\tau)}$

\section{Treatment of Four Wave Mixing Methodologies}

The previous treatment developed an exact treatment of the transients as an initial coherence evolved to final coherence under the interaction with an electromagnetic field. The treatment assumed square wave excitations that allowed a separation of driven and free induction decay components of a transition. Four wave mixing (FWM) methodologies involve three transitions that together create the output coherence but there can also be higher order processes involving more than three transitions that can also contribute. Typically, a perturbative theory ignores these higher order processes. In addition, there are multiple time orderings of the three transitions and the coherence resulting from each of these time ordered pathways must be added together to form the final coherence. In this work, we will ignore the interference effects of the multiple pathways and consider just a single pathway for each FWM methodology. This approach provides a simpler and clearer method for comparing the different methodologies. With these approximations, we derive closed form expressions for a representative pathway of each methodology. We use the driven term in our equations during the time the excitation is present and the FID term after the excitation is removed. 


\section{Transients for IR CMDS}

The excitation pulses are defined in the following figure.

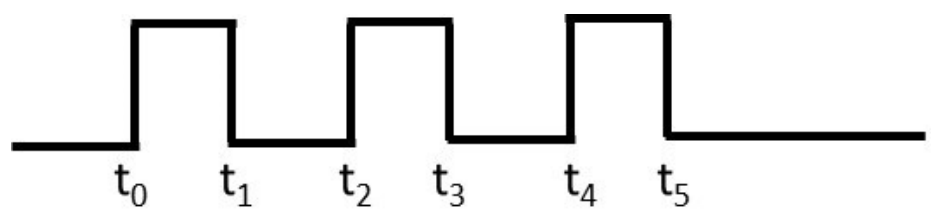

We use the previously derived relationships between the initial and subsequent coherences but we will also neglect the first term involving an initial coherence amplitude.

$\rho_{i j}=\rho_{i j}^{o} e^{-i \delta_{j i} t}+\frac{1}{2}\left(\begin{array}{l}\Omega_{i k} \tilde{\rho}_{k j}\left(\frac{e^{-i\left(\delta_{k j}+\omega\right) t}-e^{-i \delta_{i j} t}}{\left(\omega_{i k}-\omega-i\left(\Gamma_{i j}-\Gamma_{k j}\right)\right)}+\frac{e^{-i\left(\delta_{k j}-\omega\right) t}-e^{-i \delta_{j i} t}}{\left(\omega_{i k}+\omega-i\left(\Gamma_{i j}-\Gamma_{k j}\right)\right)}\right) \\ -\Omega_{j k} \tilde{\rho}_{i k}\left(\frac{e^{-i\left(\delta_{i k}+\omega\right) t}-e^{-i \delta_{i j} t}}{\left(\omega_{k j}-\omega-i\left(\Gamma_{i j}-\Gamma_{i k}\right)\right)}+\frac{e^{-i\left(\delta_{i k}-\omega\right) t}-e^{-i \delta_{i j} t}}{\left(\omega_{k j}+\omega-i\left(\Gamma_{i j}-\Gamma_{i k}\right)\right)}\right)\end{array}\right)$

At resonance, $\rho_{i j}=\rho_{i j}^{o} e^{-i \delta_{i j} t}-\frac{e^{-i \omega_{i j} t}}{2}\left(\begin{array}{l}\Omega_{i k} \tilde{\rho}_{k j}\left(\frac{e^{-\Gamma_{k j} t}-e^{-\Gamma_{i j} t}}{i\left(\Gamma_{i j}-\Gamma_{i k}\right)}+\frac{e^{-\Gamma_{k j} t}-e^{-\Gamma_{i j} t}}{i\left(\Gamma_{i j}-\Gamma_{i k}\right)}\right) \\ -\Omega_{j k} \tilde{\rho}_{i k}\left(\frac{e^{-\Gamma_{i k} t}-e^{-\Gamma_{i j} t}}{i\left(\Gamma_{i j}-\Gamma_{i k}\right)}+\frac{e^{-\Gamma_{i k} t}-e^{-\Gamma_{i j} t}}{i\left(\Gamma_{i j}-\Gamma_{i k}\right)}\right)\end{array}\right)$.

The typical pathway is $g g \stackrel{-1}{\rightarrow} g v \stackrel{2}{\rightarrow} v^{\prime} v \stackrel{3}{\rightarrow} v^{\prime} g$

gv coherence- driven

$$
\begin{aligned}
\rho_{g v}= & \rho_{g v}^{o}\left(t=t_{1}\right) e^{-i \delta_{g v}\left(t-t_{1}\right)} \Phi\left(t-t_{1}\right)+\frac{\Omega_{v g} \tilde{\rho}_{g g}}{2} \frac{e^{-\Gamma_{g g}\left(t-t_{0}\right)}-e^{-\Gamma_{g v}\left(t-t_{0}\right)}}{i\left(\Gamma_{g v}-\Gamma_{g g}\right)} e^{-i \omega_{g v}\left(t-t_{0}\right)} \\
& *\left[\Phi\left(t-t_{0}\right)-\Phi\left(t-t_{1}\right)\right]
\end{aligned}
$$

(note $\Gamma_{\mathrm{gg}}$ is zero since $\rho_{\mathrm{gg}}=1$ ).

gv coherence- FID

$$
\rho_{g v}^{o} e^{-i \delta_{g v}\left(t-t_{1}\right)}=\frac{\Omega_{v g}}{2} \frac{e^{-\Gamma_{g g}\left(t_{1}-t_{0}\right)}-e^{-\Gamma_{g v}\left(t_{1}-t_{0}\right)}}{i\left(\Gamma_{g v}-\Gamma_{g g}\right)} e^{-i \omega_{g v}\left(t_{1}-t_{0}\right)} e^{-i \delta_{g v}\left(t-t_{1}\right)} \Phi\left(t-t_{1}\right) \tilde{\rho}_{g g}
$$

(note, $\omega_{g v}=\omega_{1}-\omega_{2}$ at resonance) 
$v^{\prime} v$ coherence- driven and FID

$$
\begin{aligned}
& \rho_{v^{\prime} v}=\rho_{v^{\prime} v}^{o}\left(t=t_{3}\right) e^{-i \delta_{v^{\prime} v}\left(t-t_{3}\right)} \Phi\left(t-t_{3}\right)-\frac{\Omega_{v^{\prime} g} \tilde{\rho}_{g v}\left(t=t_{2}\right)}{2} \frac{e^{-\Gamma_{g v}\left(t-t_{2}\right)}-e^{-\Gamma_{v^{\prime} v}\left(t-t_{2}\right)}}{i\left(\Gamma_{v^{\prime} v}-\Gamma_{g v}\right)} \\
& * e^{-i \omega_{v^{\prime} v}\left(t-t_{2}\right)}\left[\Phi\left(t-t_{2}\right)-\Phi\left(t-t_{3}\right)\right] \\
& \tilde{\rho}_{g v}\left(t=t_{2}\right)=-\frac{\Omega_{v g} \tilde{\rho}_{g g}}{2} \frac{e^{-\Gamma_{g g}\left(t_{1}-t_{0}\right)}-e^{-\Gamma_{g v}\left(t_{1}-t_{0}\right)}}{i\left(\Gamma_{g v}-\Gamma_{g g}\right)} e^{-i \omega_{g v}\left(t_{1}-t_{0}\right)} e^{-i \delta_{g v}\left(t_{2}-t_{1}\right)} \\
& \rho_{v^{\prime} v}=\rho_{v^{\prime} v}^{o}\left(t=t_{3}\right) e^{-i \delta_{v^{\prime} v}\left(t-t_{3}\right)} \Phi\left(t-t_{3}\right)+\frac{\Omega_{v^{\prime} g}}{2} \frac{e^{-\Gamma_{g v}\left(t-t_{2}\right)}-e^{-\Gamma_{v^{\prime} v}\left(t-t_{2}\right)}}{i\left(\Gamma_{v^{\prime} v}-\Gamma_{g v}\right)} \\
& * e^{-i \omega_{v^{\prime} v}\left(t-t_{2}\right)} \frac{\Omega_{v g}}{2} \frac{e^{-\Gamma_{g g}\left(t_{1}-t_{0}\right)}-e^{-\Gamma_{g v}\left(t_{1}-t_{0}\right)}}{i\left(\Gamma_{g v}-\Gamma_{g g}\right)} \\
& * e^{-i \omega_{g v}\left(t_{1}-t_{0}\right)} e^{-i \delta_{g v}\left(t_{2}-t_{1}\right)} \tilde{\rho}_{g g}\left[\Phi\left(t-t_{2}\right)-\Phi\left(t-t_{3}\right)\right] \\
& \rho_{v^{\prime} v}^{o}\left(t=t_{3}\right) e^{-i \delta_{v^{\prime} v}\left(t-t_{3}\right)}=\frac{\Omega_{v^{\prime} g} \tilde{\rho}_{g v}\left(t=t_{2}\right)}{2} \frac{e^{-\Gamma_{g v}\left(t_{3}-t_{2}\right)}-e^{-\Gamma_{v^{\prime} v}\left(t_{3}-t_{2}\right)}}{i\left(\Gamma_{v^{\prime} v}-\Gamma_{g v}\right)} \\
& * e^{-i \omega_{v^{\prime} v_{v}}\left(t_{3}-t_{2}\right)} e^{-i \delta_{v^{\prime} v_{v}}\left(t-t_{3}\right)} \Phi\left(t-t_{3}\right) \\
& =-\frac{\Omega_{v^{\prime} g}}{2} \frac{e^{-\Gamma_{g v}\left(t_{3}-t_{2}\right)}-e^{-\Gamma_{v^{\prime} v}\left(t_{3}-t_{2}\right)}}{i\left(\Gamma_{v^{\prime} v}-\Gamma_{g v}\right)} e^{-i \omega_{v^{\prime} v}\left(t_{3}-t_{2}\right)} \\
& * \frac{\Omega_{v g}}{2} \frac{e^{-\Gamma_{g g}\left(t_{1}-t_{0}\right)}-e^{-\Gamma_{g v}\left(t_{1}-t_{0}\right)}}{i\left(\Gamma_{g v}-\Gamma_{g g}\right)} \\
& * e^{-i \omega_{g v}\left(t_{1}-t_{0}\right)} e^{-i \delta_{g v}\left(t_{2}-t_{1}\right)} e^{-i \delta_{v^{\prime} v}\left(t-t_{3}\right)} \tilde{\rho}_{g g} \Phi\left(t-t_{3}\right)
\end{aligned}
$$


$v^{\prime} g$ coherence

$$
\begin{aligned}
& \rho_{v^{\prime} g}=\rho_{v^{\prime} g}^{o}\left(t=t_{5}\right) e^{-i \delta_{v^{\prime} g}\left(t-t_{5}\right)} \Phi\left(t-t_{5}\right)-\frac{\Omega_{v g} \tilde{\rho}_{v^{\prime} v}\left(t=t_{4}\right)}{2} \frac{e^{-\Gamma_{v^{\prime} v}\left(t-t_{4}\right)}-e^{-\Gamma_{v^{\prime} g}\left(t-t_{4}\right)}}{i\left(\Gamma_{v^{\prime} g}-\Gamma_{v^{\prime} v}\right)} \\
& * e^{-i \omega_{v^{\prime} g}\left(t-t_{4}\right)}\left[\Phi\left(t-t_{4}\right)-\Phi\left(t-t_{5}\right)\right] \\
& \tilde{\rho}_{v^{\prime} v}\left(t=t_{4}\right)=-\frac{\Omega_{v^{\prime} g}}{2} \frac{e^{-\Gamma_{g v}\left(t_{3}-t_{2}\right)}-e^{-\Gamma_{v^{\prime} v}\left(t_{3}-t_{2}\right)}}{i\left(\Gamma_{v^{\prime} v}-\Gamma_{g v}\right)} e^{-i \omega_{v^{\prime} v}\left(t_{3}-t_{2}\right)} \\
& * \frac{\Omega_{v g}}{2} \frac{e^{-\Gamma_{g g}\left(t_{1}-t_{0}\right)}-e^{-\Gamma_{g v}\left(t_{1}-t_{0}\right)}}{i\left(\Gamma_{g v}-\Gamma_{g g}\right)} e^{-i \omega_{g v}\left(t_{1}-t_{0}\right)} e^{-i \delta_{g v}\left(t_{2}-t_{1}\right)} e^{-i \delta_{v^{\prime} v}\left(t_{4}-t_{3}\right)} \tilde{\rho}_{g g} \Phi\left(t-t_{3}\right) \\
& \rho_{v^{\prime} g}=\rho_{v^{\prime} g}^{o}\left(t=t_{5}\right) e^{-i \delta_{v^{\prime} g}\left(t-t_{5}\right)} \Phi\left(t-t_{5}\right)+\frac{\Omega_{v g} \Omega_{v^{\prime} g} \Omega_{v g}}{8} \frac{\left(e^{-\Gamma_{g v}\left(t_{3}-t_{2}\right)}-e^{-\Gamma_{v^{\prime} v}\left(t_{3}-t_{2}\right)}\right)}{i\left(\Gamma_{v^{\prime} v}-\Gamma_{g v}\right)} \\
& * \frac{\left(e^{-\Gamma_{g g}\left(t_{1}-t_{0}\right)}-e^{-\Gamma_{g v}\left(t_{1}-t_{0}\right)}\right)}{i\left(\Gamma_{g v}-\Gamma_{g g}\right)} \frac{\left(e^{-\Gamma_{v^{\prime} v}\left(t-t_{4}\right)}-e^{-\Gamma_{v^{\prime} g}\left(t-t_{4}\right)}\right)}{i\left(\Gamma_{v^{\prime} g}-\Gamma_{v^{\prime} v}\right)} \\
& * e^{-i \omega_{g v}\left(t_{2}-t_{0}\right)} e^{-i \omega_{v^{\prime} v}\left(t_{4}-t_{2}\right)} e^{-i \omega_{v^{\prime} g}\left(t-t_{4}\right)}\left[\Phi\left(t-t_{4}\right)-\Phi\left(t-t_{5}\right)\right] \\
& \tilde{\rho}_{v^{\prime} g}^{o} e^{-i \delta_{v^{\prime} g}\left(t-t_{5}\right)}=\frac{i \Omega_{v g} \Omega_{v^{\prime} g} \Omega_{v g}}{8} \\
& * \frac{\left(e^{-\Gamma_{g v}\left(t_{3}-t_{2}\right)}-e^{-\Gamma_{v^{\prime} v}\left(t_{3}-t_{2}\right)}\right)\left(e^{-\Gamma_{g g}\left(t_{1}-t_{0}\right)}-e^{-\Gamma_{g v}\left(t_{1}-t_{0}\right)}\right)\left(e^{-\Gamma_{v^{\prime} v}\left(t_{5}-t_{4}\right)}-e^{-\Gamma_{v^{\prime} g}\left(t_{5}-t_{4}\right)}\right)}{\left(\Gamma_{v^{\prime} v}-\Gamma_{g v}\right)\left(\Gamma_{g v}-\Gamma_{g g}\right)\left(\Gamma_{v^{\prime} g}-\Gamma_{v^{\prime} v}\right)} \\
& * e^{-i \omega_{g v}\left(t_{2}-t_{0}\right)} e^{-i \omega_{v^{\prime} v}\left(t_{4}-t_{2}\right)} e^{-i \omega_{v^{\prime} g}\left(t-t_{4}\right)} e^{-\Gamma_{v^{\prime} g}\left(t-t_{5}\right)} \Phi\left(t-t_{5}\right)
\end{aligned}
$$




\section{Transients for Vibrational Pump-Probe CMDS}

Typical Pathway $g g \stackrel{-1}{\rightarrow} g{ }^{\stackrel{2}{\rightarrow}} v^{\prime} v \stackrel{3}{\rightarrow} v^{\prime} g$

gv coherence

$\rho_{g v}=\rho_{g v}^{o}\left(t=t_{1}\right) e^{-i \delta_{g v}\left(t-t_{1}\right)} \Phi\left(t-t_{1}\right)+\frac{\Omega_{v g} \tilde{\rho}_{g g}}{2} \frac{e^{-\Gamma_{g g} t}-e^{-\Gamma_{g v} t}}{i\left(\Gamma_{g v}-\Gamma_{g g}\right)} e^{-i \omega_{g v} t}\left[\Phi\left(t-t_{0}\right)-\Phi\left(t-t_{1}\right)\right] \quad$ (note $\Gamma_{g g}$ is zero since $\left.\rho_{g g}=1\right)$

$$
\rho_{g v}^{o} e^{-i \delta_{g v}\left(t-t_{1}\right)}=\frac{\Omega_{v g}}{2} \frac{e^{-\Gamma_{g g} t}-e^{-\Gamma_{g v} t}}{i\left(\Gamma_{g v}-\Gamma_{g g}\right)} e^{-i \omega_{g v}\left(t_{1}-t_{0}\right)} e^{-i \delta_{g v}\left(t-t_{1}\right)} \Phi\left(t-t_{1}\right) \tilde{\rho}_{g g} \quad \text { (note, } \omega_{g v}=\omega_{1}-\omega_{2} \text { at resonance) }
$$

$v v$ population

$$
\begin{aligned}
& \rho_{v v}=\rho_{v v}^{o}\left(t=t_{3}\right) e^{-i \Gamma_{v v}\left(t-t_{3}\right)} \Phi\left(t-t_{3}\right)-\frac{\Omega_{v^{\prime} g} \tilde{\rho}_{g v}(t)}{2} \frac{e^{-\Gamma_{g, t} t}-e^{-\Gamma_{v^{\prime},}, t}}{i\left(\Gamma_{v^{\prime} v}-\Gamma_{g v}\right)} e^{-i \omega_{v^{\prime} v^{\prime}} t}\left[\Phi\left(t-t_{2}\right)-\Phi\left(t-t_{3}\right)\right] \\
& \tilde{\rho}_{g v}(t)=-\frac{\Omega_{v g} \tilde{\rho}_{g g}}{2} \frac{e^{-\Gamma_{g g} t}-e^{-\Gamma_{g v} t}}{i\left(\Gamma_{g v}-\Gamma_{g g}\right)} \\
& \rho_{v v}=\rho_{v v}^{o}\left(t=t_{3}\right) e^{-i \delta_{v v}\left(t-t_{3}\right)} \Phi\left(t-t_{3}\right)+\frac{\Omega_{v g}}{2} \frac{e^{-\Gamma_{g v} t}-e^{-\Gamma_{v v} t}}{i\left(\Gamma_{v v}-\Gamma_{g v}\right)} \frac{\Omega_{v g}}{2} \frac{e^{-\Gamma_{g g} t}-e^{-\Gamma_{g v} t}}{i\left(\Gamma_{g v}-\Gamma_{g g}\right)} e^{-i \omega_{v v} t} \tilde{\rho}_{g g}\left[\Phi\left(t-t_{2}\right)-\Phi\left(t-t_{3}\right)\right] \\
& \rho_{v v}^{o}\left(t=t_{3}\right) e^{-i \delta_{v v}\left(t-t_{3}\right)}=\frac{\Omega_{v g} \tilde{\rho}_{g v}\left(t=t_{2}\right)}{2} \frac{e^{-\Gamma_{g v}\left(t_{3}-t_{2}\right)}-e^{-\Gamma_{v v}\left(t_{3}-t_{2}\right)}}{i\left(\Gamma_{v v}-\Gamma_{g v}\right)} e^{-i \omega_{v v}\left(t_{3}-t_{2}\right)} e^{-i \delta_{w_{v}}\left(t-t_{3}\right)} \Phi\left(t-t_{3}\right) \\
& =-\frac{\Omega_{v g}^{2}}{4} \frac{e^{-\Gamma_{g v}\left(t_{3}-t_{2}\right)}-e^{-\Gamma_{w}\left(t_{3}-t_{2}\right)}}{i\left(\Gamma_{v v}-\Gamma_{g v}\right)} \frac{e^{-\Gamma_{g g}\left(t_{1}-t_{0}\right)}-e^{-\Gamma_{g v}\left(t_{1}-t_{0}\right)}}{i\left(\Gamma_{g v}-\Gamma_{g g}\right)} e^{-i \omega_{v v}\left(t_{3}-t_{2}\right)} e^{-i \delta_{g v}\left(t_{2}-t_{1}\right)} e^{-i \delta_{v v}\left(t-t_{3}\right)} \tilde{\rho}_{g g} \Phi\left(t-t_{3}\right)
\end{aligned}
$$

$v g$ coherence

$$
\begin{aligned}
& \rho_{v g}=\rho_{v g}^{o}\left(t=t_{5}\right) e^{-i \delta_{v g}\left(t-t_{5}\right)} \Phi\left(t-t_{5}\right)-\frac{\Omega_{v g} \tilde{\rho}_{v^{\prime} v}\left(t=t_{4}\right)}{2} \frac{e^{-\Gamma_{v v}\left(t-t_{4}\right)}-e^{-\Gamma_{v g}\left(t-t_{4}\right)}}{i\left(\Gamma_{v g}-\Gamma_{v v}\right)} \\
& * e^{-i \omega_{\text {rg }}\left(t-t_{4}\right)}\left[\Phi\left(t-t_{4}\right)-\Phi\left(t-t_{5}\right)\right] \\
& \tilde{\rho}_{v v}\left(t=t_{4}\right)=-\frac{\Omega_{v g}^{2}}{4} \frac{e^{-\Gamma_{g v}\left(t_{3}-t_{2}\right)}-e^{-\Gamma_{v v}\left(t_{3}-t_{2}\right)}}{i\left(\Gamma_{v v}-\Gamma_{g v}\right)} e^{-i \omega_{v v} v\left(t_{3}-t_{2}\right)} \\
& * \frac{e^{-\Gamma_{g g}\left(t_{1}-t_{0}\right)}-e^{-\Gamma_{g v}\left(t_{1}-t_{0}\right)}}{i\left(\Gamma_{g v}-\Gamma_{g g}\right)} e^{-i \omega_{g v}\left(t_{1}-t_{0}\right)} e^{-i \delta_{g v}\left(t_{2}-t_{1}\right)} e^{-i \delta_{w v}\left(t_{4}-t_{3}\right)} \tilde{\rho}_{g g} \Phi\left(t-t_{3}\right) \\
& \rho_{v g}=\rho_{v^{\prime} g}^{o}\left(t=t_{5}\right) e^{-i \delta_{v g}\left(t-t_{5}\right)} \Phi\left(t-t_{5}\right)+\frac{\Omega_{v g}^{3}}{8} \frac{\left(e^{-\Gamma_{g v}\left(t_{3}-t_{2}\right)}-e^{-\Gamma_{v}\left(t_{3}-t_{2}\right)}\right)}{i\left(\Gamma_{v v}-\Gamma_{g v}\right)} \\
& * \frac{\left(e^{-\Gamma_{g g}\left(t_{1}-t_{0}\right)}-e^{-\Gamma_{g v}\left(t_{1}-t_{0}\right)}\right)}{i\left(\Gamma_{g v}-\Gamma_{g g}\right)} \frac{\left(e^{-\Gamma_{v v}\left(t-t_{4}\right)}-e^{-\Gamma_{v g}\left(t-t_{4}\right)}\right)}{i\left(\Gamma_{v g}-\Gamma_{v v}\right)} \\
& * e^{-i \omega_{g v}\left(t_{2}-t_{0}\right)} e^{-i \omega_{v^{\prime} v_{v}\left(t_{4}-t_{2}\right)}} e^{-i \omega_{v^{\prime} g}\left(t-t_{4}\right)}\left[\Phi\left(t-t_{4}\right)-\Phi\left(t-t_{5}\right)\right] \\
& \tilde{\rho}_{v g}^{o} e^{-i \delta_{v g}\left(t-t_{5}\right)}=\frac{i \Omega_{v g}^{3}}{8} \\
& * \frac{\left(e^{-\Gamma_{g v}\left(t_{3}-t_{2}\right)}-e^{-\Gamma_{v^{\prime} v}\left(t_{3}-t_{2}\right)}\right)\left(e^{-\Gamma_{g g}\left(t_{1}-t_{0}\right)}-e^{-\Gamma_{g v}\left(t_{1}-t_{0}\right)}\right)\left(e^{-\Gamma_{v v}\left(t_{5}-t_{4}\right)}-e^{-\Gamma_{v g}\left(t_{5}-t_{4}\right)}\right)}{\left(\Gamma_{v v}-\Gamma_{g v}\right)\left(\Gamma_{g v}-\Gamma_{g g}\right)\left(\Gamma_{v g}-\Gamma_{v^{\prime} v}\right)} \\
& * e^{-i \omega_{g v}\left(t_{2}-t_{0}\right)} e^{-i \omega_{w}\left(t_{4}-t_{2}\right)} e^{-i \omega_{\text {tg }}\left(t-t_{4}\right)} e^{-\Gamma_{\text {vg }}\left(t-t_{5}\right)} \Phi\left(t-t_{5}\right)
\end{aligned}
$$




\section{Transients for DOVE CMDS}

Typical pathway $g g \rightarrow g v^{\prime} \rightarrow\left(v+v^{\prime}\right), v^{\prime} \rightarrow\left(e+v^{\prime}\right), v^{\prime}$

$g v^{\prime}$ coherence from $g g$

$$
\begin{gathered}
\rho_{g v^{\prime}}=\rho_{g v^{\prime}}^{o}\left(t=t_{1}\right) e^{-i \delta_{g v^{\prime}}\left(t-t_{1}\right)} \Phi\left(t-t_{1}\right)+\frac{\Omega_{v^{\prime} g} g \tilde{\rho}_{g g}}{2} \frac{e^{-\Gamma_{g g^{\prime}} t}-e^{-\Gamma_{g v^{\prime}} t}}{i\left(\Gamma_{g v^{\prime}}-\Gamma_{g g}\right)} e^{-i \omega_{g g^{\prime}} t}\left(\Phi\left(t-t_{0}\right)-\Phi\left(t-t_{1}\right)\right) \\
\rho_{g v^{\prime}}^{o} e^{-i \delta_{g^{\prime}}\left(t-t_{1}\right)}=\frac{\Omega_{v^{\prime} g}}{2} \frac{e^{-\Gamma_{g g}\left(t_{1}-t_{0}\right)}-e^{-\Gamma_{g v^{\prime}}\left(t_{1}-t_{0}\right)}}{i\left(\Gamma_{g v^{\prime}}-\Gamma_{g g}\right)} e^{-i \delta_{g v^{\prime}}\left(t-t_{0}\right)} \Phi\left(t-t_{1}\right) \tilde{\rho}_{g g}
\end{gathered}
$$

$\left(v+v^{\prime}\right), v^{\prime}$ coherence

$$
\begin{aligned}
& \rho_{\left(v+v^{\prime}\right), v^{\prime}}=\rho_{\left(v+v^{\prime}\right), v^{\prime}}^{o}\left(t=t_{3}\right) e^{-i \delta_{(v+v), v^{\prime}}\left(t-t_{3}\right)} \Phi\left(t-t_{3}\right)-\frac{\Omega_{\left(v+v^{\prime}\right), g} \tilde{\rho}_{g v^{\prime}}\left(t=t_{2}\right)}{2} \frac{e^{-\Gamma_{g v^{\prime}}\left(t-t_{2}\right)}-e^{-\Gamma_{(v+v), v^{\prime}}\left(t-t_{2}\right)}}{i\left(\Gamma_{\left(v+v^{\prime}\right), v^{\prime}}-\Gamma_{g v^{\prime}}\right)}
\end{aligned}
$$

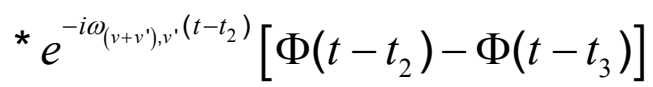

$$
\begin{aligned}
& \tilde{\rho}_{g v^{\prime}}\left(t=t_{2}\right)=-\frac{\Omega_{v^{\prime} g} \tilde{\rho}_{g g}}{2} \frac{e^{-\Gamma_{g g}\left(t_{1}-t_{0}\right)}-e^{-\Gamma_{g v^{\prime}}\left(t_{1}-t_{0}\right)}}{i\left(\Gamma_{g v^{\prime}}-\Gamma_{g g}\right)} e^{-i \delta_{g v^{\prime}}\left(t_{2}-t_{0}\right)} \\
& \rho_{\left(v+v^{\prime}\right), v^{\prime}}=\rho_{\left(v+v^{\prime}\right), v^{\prime}}^{o}\left(t=t_{3}\right) e^{-i \delta_{(v+v), v^{\prime}}\left(t-t_{3}\right)} \Phi\left(t-t_{3}\right)+\frac{\Omega_{\left(v+v^{\prime}\right), g}}{2} \frac{e^{-\Gamma_{g v^{\prime}}\left(t-t_{2}\right)}-e^{-\Gamma_{\left(v+v^{\prime}\right) v^{\prime}\left(t-t_{2}\right)}}}{i\left(\Gamma_{\left(v+v^{\prime}\right), v^{\prime}}-\Gamma_{g v^{\prime}}\right)} \\
& * e^{-i \omega_{(v+t), v^{\prime},\left(t-t_{2}\right)}} \frac{\Omega_{v^{\prime} g}}{2} \frac{e^{-\Gamma_{g g}\left(t_{1}-t_{0}\right)}-e^{-\Gamma_{g v^{\prime}}\left(t_{1}-t_{0}\right)}}{i\left(\Gamma_{g v^{\prime}}-\Gamma_{g g}\right)} e^{-i \delta_{g v^{\prime}}\left(t_{2}-t_{0}\right)} \tilde{\rho}_{g g}\left[\Phi\left(t-t_{2}\right)-\Phi\left(t-t_{3}\right)\right] \\
& \rho_{\left(v+v^{\prime}\right), v^{\prime}}^{o}\left(t=t_{3}\right) e^{-i \delta_{(v+v) v^{\prime}, v^{\prime}\left(t-t_{3}\right)}^{o}}=\frac{\Omega_{\left(v+v^{\prime}\right), g} \tilde{\rho}_{g v^{\prime}}\left(t=t_{2}\right)}{2} \frac{e^{-\Gamma_{g v^{\prime}}\left(t_{3}-t_{2}\right)}-e^{-\Gamma_{\left(v+v^{\prime}\right), v^{\prime}}\left(t_{3}-t_{2}\right)}}{i\left(\Gamma_{v^{\prime} v}-\Gamma_{g v^{\prime}}\right)}
\end{aligned}
$$

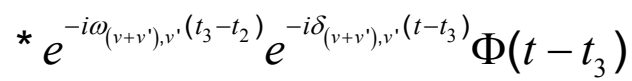

$$
\begin{aligned}
& =-\frac{\Omega_{v^{\prime} g} \Omega_{\left(v+v^{\prime}\right), g}}{4} \frac{e^{-\Gamma_{g^{\prime}}\left(t_{3}-t_{2}\right)}-e^{\left.-\Gamma_{\left(v+v v^{\prime} v^{\prime} t_{3}\right.} t_{3}-t_{2}\right)}}{i\left(\Gamma_{\left(v+v^{\prime}\right), v^{\prime}}-\Gamma_{g v^{\prime}}\right)} \frac{e^{-\Gamma_{g g}\left(t_{1}-t_{0}\right)}-e^{-\Gamma_{g v^{\prime}}\left(t_{1}-t_{0}\right)}}{i\left(\Gamma_{g v^{\prime}}-\Gamma_{g g}\right)}
\end{aligned}
$$

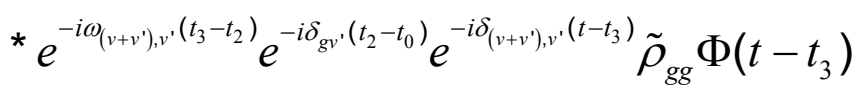


$\left(e+v^{\prime}\right) v^{\prime}$ coherence from $\left(v+v^{\prime}\right), v^{\prime}$.

Note that fast dephasing of $\left(e+v^{\prime}\right), v^{\prime}$ eliminates its FID contribution.

Only the FID from the $\left(v+v^{\prime}\right), v^{\prime}$ feeding coherence is important

$$
\begin{aligned}
& \rho_{\left(e+v^{\prime}\right) v^{\prime}}=\frac{\Omega_{\left(v+v^{\prime}\right),\left(e+v^{\prime}\right)} \tilde{\rho}_{\left(v+v^{\prime}\right), v^{\prime}}\left(t-t_{4}\right)}{2} \frac{e^{-i\left(\omega_{\left(v+v^{\prime}\right), v^{\prime}}-i \Gamma_{\left(v+v^{\prime}\right), v^{\prime}}+\omega_{3}\right)\left(t-t_{2}\right)}}{\left(\omega_{\left(e+v^{\prime}\right), v^{\prime}}-\omega_{3}-i\left(\Gamma_{\left(e+v^{\prime}\right), v^{\prime}}-\Gamma_{\left(v+v^{\prime}\right), v^{\prime}}\right)\right)} \\
& *\left(\Phi\left(t-t_{4}\right)-\Phi\left(t-t_{5}\right)\right) \\
& \tilde{\rho}_{\left(v+v^{\prime}\right), v^{\prime}}\left(t=t_{4}\right) e^{-i \delta_{\left(v+v^{\prime}\right), v^{\prime}}\left(t-t_{3}\right)}=-\frac{\Omega_{v^{\prime} g} \Omega_{\left(v+v^{\prime}\right), g}}{4} \frac{e^{-\Gamma_{g v^{\prime}}\left(t_{3}-t_{2}\right)}-e^{-\Gamma_{\left(v+v^{\prime}, v^{\prime}\right.}\left(t_{3}-t_{2}\right)}}{i\left(\Gamma_{\left(v+v^{\prime}\right), v^{\prime}}-\Gamma_{g v^{\prime}}\right)} \\
& * \frac{e^{-\Gamma_{g g}\left(t_{1}-t_{0}\right)}-e^{-\Gamma_{g v^{\prime}}\left(t_{1}-t_{0}\right)}}{i\left(\Gamma_{g v^{\prime}}-\Gamma_{g g}\right)} e^{-i \omega_{\left(v+v^{\prime}\right) v^{\prime}}\left(t_{3}-t_{2}\right)} e^{-i \delta_{g v^{\prime}}\left(t_{2}-t_{0}\right)} e^{-i \delta_{\left(v+v^{\prime}\right), v^{\prime}\left(t_{4}-t_{3}\right)}} \tilde{\rho}_{g g} \Phi\left(t-t_{3}\right) \\
& \rho_{\left(e+v^{\prime}\right) v^{\prime}}=-\frac{\Omega_{v^{\prime} g} \Omega_{\left(v+v^{\prime}\right), g} \Omega_{\left(v+v^{\prime}\right),\left(e+v^{\prime}\right)}}{8} \frac{e^{-i\left(\omega_{\left(v+v^{\prime}\right), v^{\prime}}-i \Gamma_{\left(v+v^{\prime}\right), v^{\prime}}+\omega_{3}\right)\left(t-t_{2}\right)}}{\left(\omega_{\left(e+v^{\prime}\right), v^{\prime}}-\omega_{3}\right)} \frac{e^{-\Gamma_{g v^{\prime}}\left(t_{3}-t_{2}\right)}-e^{-\Gamma_{\left(v+v^{\prime}\right), v^{\prime}}\left(t_{3}-t_{2}\right)}}{i\left(\Gamma_{\left(v+v^{\prime}\right), v^{\prime}}-\Gamma_{g v^{\prime}}\right)} \\
& * \frac{e^{-\Gamma_{g g}\left(t_{1}-t_{0}\right)}-e^{-\Gamma_{g v^{\prime}}\left(t_{1}-t_{0}\right)}}{i\left(\Gamma_{g v^{\prime}}-\Gamma_{g g}\right)} e^{-i \omega_{\left(v+v^{\prime}\right), v^{\prime}}\left(t_{3}-t_{2}\right)} e^{-i \delta_{g v^{\prime}}\left(t_{2}-t_{0}\right)} e^{-i \delta_{\left(v+v^{\prime}, v^{\prime}\right.}\left(t_{4}-t_{3}\right)} \tilde{\rho}_{g g}\left(\Phi\left(t-t_{4}\right)-\Phi\left(t-t_{5}\right)\right)
\end{aligned}
$$




\section{Transients for TRSF CMDS}

Typical Pathway $g g \rightarrow v g \rightarrow\left(v+v^{\prime}\right), g \rightarrow(e+v), g$ $v g$ coherence from $g g$

$$
\begin{gathered}
\rho_{v g}=\rho_{v g}^{o}\left(t=t_{1}\right) e^{-i \delta_{g g}\left(t-t_{1}\right)} \Phi\left(t-t_{1}\right)+\frac{\Omega_{v g} \tilde{\rho}_{g g}}{2} \frac{e^{-\Gamma_{g g} t}-e^{-\Gamma_{v g} t}}{i\left(\Gamma_{v g}-\Gamma_{g g}\right)} e^{-i \omega_{v g} t}\left(\Phi\left(t-t_{0}\right)-\Phi\left(t-t_{1}\right)\right) \\
\rho_{v g}^{o} e^{-i \delta_{v g}\left(t-t_{1}\right)}=\frac{\Omega_{v g}}{2} \frac{e^{-\Gamma_{g g}\left(t_{1}-t_{0}\right)}-e^{-\Gamma_{v g}\left(t_{1}-t_{0}\right)}}{i\left(\Gamma_{v g}-\Gamma_{g g}\right)} e^{-i \delta_{v g}\left(t-t_{0}\right)} \Phi\left(t-t_{1}\right) \tilde{\rho}_{g g}
\end{gathered}
$$

$\left(v+v^{\prime}\right), g$ coherence

$$
\begin{aligned}
& \rho_{\left(v+v^{\prime}\right), g}=\rho_{\left(v+v^{\prime}\right), g}^{o}\left(t=t_{3}\right) e^{-i \delta_{(v+v), g}\left(t-t_{3}\right)} \Phi\left(t-t_{3}\right)-\frac{\Omega_{\left(v+v^{\prime}\right), v} \tilde{\rho}_{v g}\left(t=t_{2}\right)}{2} \frac{e^{-\Gamma_{v g}\left(t-t_{2}\right)}-e^{\left.-\Gamma_{(v+v)}\right) g}\left(t-t_{2}\right)}{i\left(\Gamma_{\left(v+v^{\prime}\right), g}-\Gamma_{v g}\right)}
\end{aligned}
$$

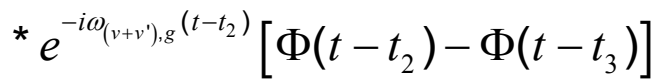

$$
\begin{aligned}
& \tilde{\rho}_{v g}\left(t=t_{2}\right)=-\frac{\Omega_{v g} \tilde{\rho}_{g g}}{2} \frac{e^{-\Gamma_{g g}\left(t_{1}-t_{0}\right)}-e^{-\Gamma_{v g}\left(t_{1}-t_{0}\right)}}{i\left(\Gamma_{v g}-\Gamma_{g g}\right)} e^{-i \delta_{v g}\left(t_{2}-t_{0}\right)} \\
& \rho_{\left(v+v^{\prime}\right), g}=\rho_{\left(v+v^{\prime}\right), g}^{o}\left(t=t_{3}\right) e^{-i \delta_{(v+v), g}\left(t-t_{3}\right)} \Phi\left(t-t_{3}\right)+\frac{\Omega_{\left(v+v^{\prime}\right), v}}{2} \frac{e^{-\Gamma_{v g}\left(t-t_{2}\right)}-e^{-\Gamma_{\left(v+v^{\prime}\right), g}\left(t-t_{2}\right)}}{i\left(\Gamma_{\left(v+v^{\prime}\right), g}-\Gamma_{v g}\right)} \\
& * e^{-i \omega_{(v+t))_{g}\left(t-t_{2}\right)}} \frac{\Omega_{v g}}{2} \frac{e^{-\Gamma_{g g}\left(t_{1}-t_{0}\right)}-e^{-\Gamma_{v g}\left(t_{1}-t_{0}\right)}}{i\left(\Gamma_{v g}-\Gamma_{g g}\right)} e^{-i \delta_{g g}\left(t_{2}-t_{0}\right)} \tilde{\rho}_{g g}\left[\Phi\left(t-t_{2}\right)-\Phi\left(t-t_{3}\right)\right] \\
& \rho_{\left(v+v^{\prime}\right), g}^{o}\left(t=t_{3}\right) e^{-i \delta_{\left(v+v^{\prime}\right), g}\left(t-t_{3}\right)}=\frac{\Omega_{\left(v+v^{\prime}\right), v} \tilde{\rho}_{g v^{\prime}}\left(t=t_{2}\right)}{2} \frac{e^{-\Gamma_{v g}\left(t_{3}-t_{2}\right)}-e^{\left.-\Gamma_{(v+v)}\right), g\left(t_{3}-t_{2}\right)}}{i\left(\Gamma_{v^{\prime} v}-\Gamma_{g v^{\prime}}\right)} \\
& * e^{-i \omega_{(t+v), g}\left(t_{3}-t_{2}\right)} e^{-i \delta_{(t+v), g}\left(t-t_{3}\right)} \Phi\left(t-t_{3}\right) \\
& =-\frac{\Omega_{v g} \Omega_{\left(v+v^{\prime}\right), v}}{4} \frac{e^{-\Gamma_{v g}\left(t_{3}-t_{2}\right)}-e^{\left.-\Gamma_{(v+v)}\right) g\left(t_{3}-t_{2}\right)}}{i\left(\Gamma_{\left(v+v^{\prime}\right), g}-\Gamma_{v g}\right)} \frac{e^{-\Gamma_{g g}\left(t_{1}-t_{0}\right)}-e^{-\Gamma_{g v^{\prime}}\left(t_{1}-t_{0}\right)}}{i\left(\Gamma_{v g}-\Gamma_{g g}\right)} \\
& * e^{\left.-i \omega_{(v+v)}\right) g\left(t_{3}-t_{2}\right)} e^{-i \delta_{\delta_{g}}\left(t_{2}-t_{0}\right)} e^{-i \delta_{(t+v) \cdot g}\left(t-t_{3}\right)} \tilde{\rho}_{g g} \Phi\left(t-t_{3}\right)
\end{aligned}
$$


$(e+v), g$ coherence from $\left(v+v^{\prime}\right), g$.

Note that fast dephasing of $(e+v), g$ eliminates its FID contribution.

Only the FID from the $\left(v+v^{\prime}\right), g$ feeding coherence is important

$$
\begin{aligned}
& \rho_{(e+v), g}=\frac{\Omega_{\left(v+v^{\prime}\right),(e+v)} \tilde{\rho}_{\left(v+v^{\prime}\right), g}\left(t-t_{4}\right)}{2} \frac{e^{-i\left(\omega_{\left(v+v^{\prime}\right), g}-i \Gamma_{\left(v+v^{\prime}\right), g}+\omega_{3}\right)\left(t-t_{2}\right)}}{\left(\omega_{\left(e+v^{\prime}\right), g}-\omega_{3}-i\left(\Gamma_{\left(e+v^{\prime}\right), g}-\Gamma_{\left(v+v^{\prime}\right), g}\right)\right)} \\
& *\left(\Phi\left(t-t_{4}\right)-\Phi\left(t-t_{5}\right)\right) \\
& \tilde{\rho}_{\left(v+v^{\prime}\right), g}\left(t=t_{4}\right) e^{-i \delta_{\left(v+v^{\prime}\right), g}\left(t-t_{3}\right)}=-\frac{\Omega_{v g} \Omega_{\left(v+v^{\prime}\right), v}}{4} \frac{e^{-\Gamma_{v g}\left(t_{3}-t_{2}\right)}-e^{-\Gamma_{\left(v+v^{\prime}\right), g}\left(t_{3}-t_{2}\right)}}{i\left(\Gamma_{\left(v+v^{\prime}\right), g}-\Gamma_{v g}\right)} \\
& * \frac{e^{-\Gamma_{g g}\left(t_{1}-t_{0}\right)}-e^{-\Gamma_{v g}\left(t_{1}-t_{0}\right)}}{i\left(\Gamma_{v g}-\Gamma_{g g}\right)} e^{-i \omega_{\left.(v+v)^{\prime}\right), g}\left(t_{3}-t_{2}\right)} e^{-i \delta_{v g}\left(t_{2}-t_{0}\right)} e^{-i \delta_{\left(v+v^{\prime}\right) g}\left(t_{4}-t_{3}\right)} \tilde{\rho}_{g g} \Phi\left(t-t_{3}\right) \\
& \rho_{\left(e+v^{\prime}\right) g}=-\frac{\Omega_{v g} \Omega_{\left(v+v^{\prime}\right), v} \Omega_{\left(v+v^{\prime}\right),(e+v)}}{8} \frac{e^{-i\left(\omega_{\left(v+v^{\prime}\right), g}-i \Gamma_{\left(v+v^{\prime}\right), g}+\omega_{3}\right)\left(t-t_{2}\right)}}{\left(\omega_{(e+v), g}-\omega_{3}\right)} \frac{e^{-\Gamma_{g v^{\prime}}\left(t_{3}-t_{2}\right)}-e^{-\Gamma_{\left(v+v^{\prime}\right), v^{\prime}}\left(t_{3}-t_{2}\right)}}{i\left(\Gamma_{\left(v+v^{\prime}\right), g}-\Gamma_{v g}\right)} \\
& * \frac{e^{-\Gamma_{g g}\left(t_{1}-t_{0}\right)}-e^{-\Gamma_{v g}\left(t_{1}-t_{0}\right)}}{i\left(\Gamma_{v g}-\Gamma_{g g}\right)} e^{-i \omega_{\left(v+v^{\prime}\right) \cdot g}\left(t_{3}-t_{2}\right)} e^{-i \delta_{v g}\left(t_{2}-t_{0}\right)} e^{-i \delta_{\left(v+v^{\prime}\right) . g}\left(t_{4}-t_{3}\right)} \tilde{\rho}_{g g}\left(\Phi\left(t-t_{4}\right)-\Phi\left(t-t_{5}\right)\right)
\end{aligned}
$$




\section{Transients for CARS}

The CARS pathway is $g g \rightarrow e g \rightarrow v g \rightarrow e g$

Note that fast dephasing of the eg coherences eliminates their FID contribution. Only the FID from the $v g$ feeding coherence is important.

eg coherence from $g g$

$\rho_{e g}=\frac{\Omega_{e g} \tilde{\rho}_{g g}}{2\left(\omega_{e g}-\omega\right)} e^{-i \omega_{1}\left(t-t_{0}\right)}\left(\Phi\left(t-t_{0}\right)-\Phi\left(t-t_{1}\right)\right)$

$v g$ coherence from eg assuming temporal overlap with eg

$$
\begin{aligned}
\rho_{v g}= & \frac{i \Omega_{v e} \tilde{\rho}_{e g}\left(1-e^{-\Gamma_{v g}\left(t-t_{0}\right)}\right)}{2 \Gamma_{v g}} e^{-i\left(\omega_{1}-\omega_{2}\right)\left(t-t_{0}\right)} \\
= & \frac{i \Omega_{v e}\left(1-e^{-\Gamma_{v g}\left(t-t_{0}\right)}\right)}{2 \Gamma_{v g}} e^{-i\left(\omega_{1}-\omega_{2}\right)\left(t-t_{0}\right) *} \frac{\Omega_{e g} \tilde{\rho}_{g g}}{2\left(\omega_{e g}-\omega\right)} e^{-i \omega_{1}\left(t-t_{0}\right)}\left(\Phi\left(t-t_{0}\right)-\Phi\left(t-t_{1}\right)\right) \\
& +\frac{i \Omega_{v e}\left(1-e^{-\Gamma_{v g}\left(t_{1}-t_{0}\right)}\right)}{2 \Gamma_{v g}} e^{-i\left(\omega_{1}-\omega_{2}\right)\left(t_{1}-t_{0}\right) *} \frac{\Omega_{e g} \tilde{\rho}_{g g}}{2\left(\omega_{e g}-\omega\right)} e^{-i \omega_{1}\left(t_{1}-t_{0}\right) *} e^{-i\left(\omega_{v g}-i \Gamma_{v g}\right)\left(t-t_{1}\right)} \Phi\left(t-t_{1}\right)
\end{aligned}
$$

eg coherence from $v g$. Dephasing eliminates FID

$$
\begin{aligned}
\rho_{e g} & =\frac{\Omega_{e v} \tilde{\rho}_{v g}}{2} \frac{e^{-i\left(\omega_{v g}-i \Gamma_{v g}+\omega_{3}\right)\left(t-t_{2}\right)}}{\left(\omega_{e v}-\omega_{3}-i\left(\Gamma_{e g}-\Gamma_{v g}\right)\right)} \\
= & \frac{\Omega_{e v}}{2} \frac{e^{-i\left(\omega_{g g}-i \Gamma_{v g}+\omega_{3}\right)\left(t-t_{2}\right)}}{\left(\omega_{e v}-\omega_{3}\right)}\left(\Phi\left(t-t_{2}\right)-\Phi\left(t-t_{3}\right)\right) \\
& * \frac{i \Omega_{v e}\left(1-e^{-\Gamma_{g g}\left(t_{1}-t_{0}\right)}\right)}{2 \Gamma_{v g}} e^{-i\left(\omega_{1}-\omega_{2}\right)\left(t_{1}-t_{0}\right)} * \frac{\Omega_{e g} \tilde{\rho}_{g g}}{2\left(\omega_{e g}-\omega\right)} e^{-i \omega_{1}\left(t_{1}-t_{0}\right) *} e^{-i\left(\omega_{v g}-i \Gamma_{r g}\right)\left(t-t_{2}\right)}
\end{aligned}
$$




\section{Comparison of spectral scans across a resonance using a rectangular or Gaussian excitation pulse.}

The top row shows the spectral shapes of a rectangular and Gaussian excitation pulse for three different pulse widths, $\Delta t$. It also shows the Fourier transform of the free induction decay of a coherence with a 1 ps dephasing time. The bottom row shows the spectrum that results from scanning the frequency of the excitation pulse across the resonance frequency, $\bar{v}_{i j}$, of the $\rho_{i j}$ coherence. The sharp cut-on/cut-off of the rectangular excitation pulse results in the weak fringes expected for a sinc function.
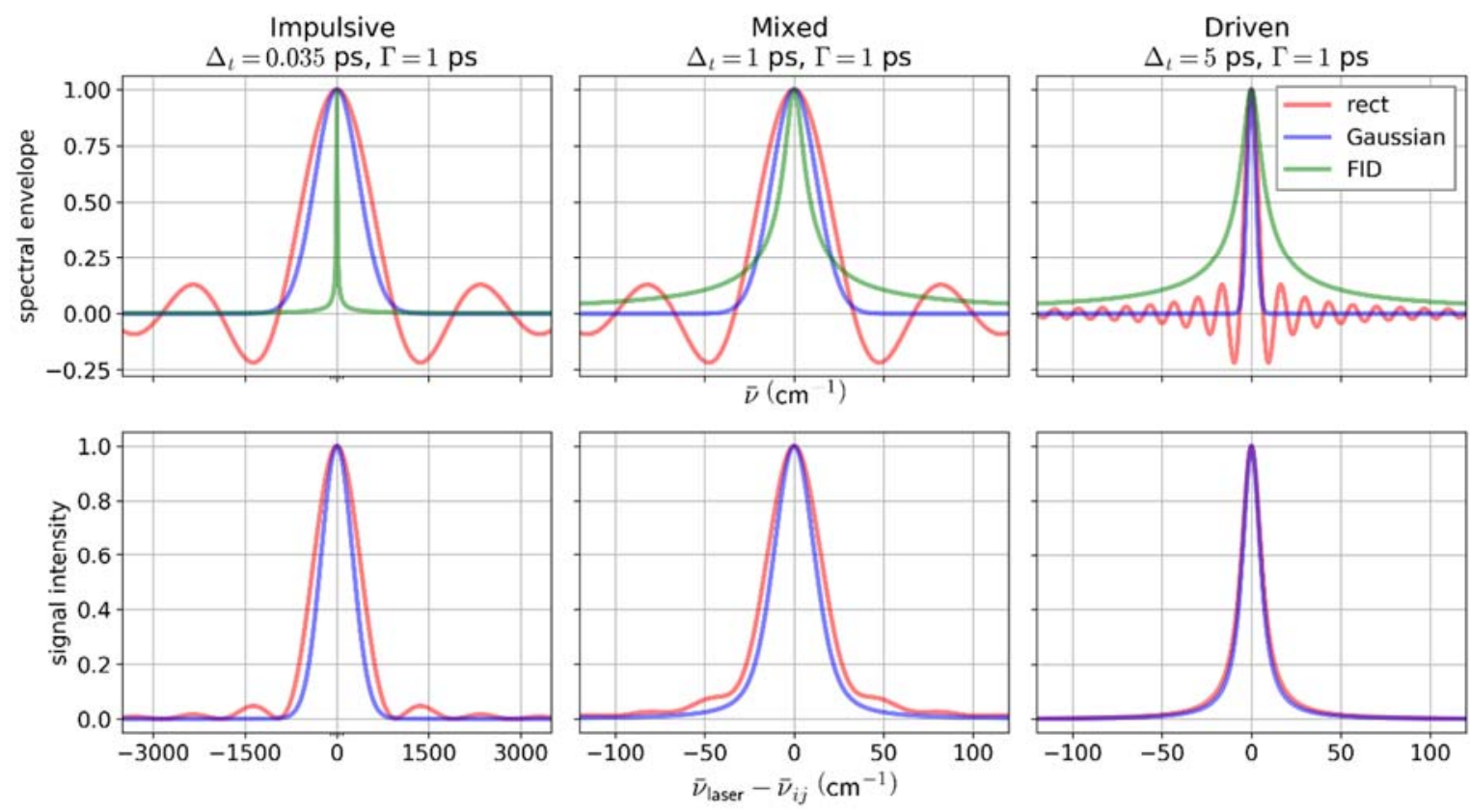

Derivation of the spectra for Heaviside and Gaussian pulse envelopes

The Liouville Equation in the rotating frame, $\omega_{i j}$ is

$\dot{\rho}_{i j}=-\Gamma \rho_{i j}+q(t) e^{i \Omega t}$

where $\Omega \equiv \pm \omega_{\text {laser }}-\omega_{i j}$ is the detuning from resonance and $\mathrm{q}(\mathrm{t})$ has the temporal dependence of the pulse envelope. The general solution is

$\rho_{i j}(t)=\int_{-\infty}^{\infty} q(t-u) e^{i \Omega(t-u)} e^{-\Gamma u} \Theta(u) d u$

in the time domain and

$P_{i j}(\omega)=\frac{Q(\omega-\Omega)}{\Gamma+i \omega}$

in the frequency domain. Here $P_{i j}$ and $Q$ are the Fourier transforms of $\rho_{\mathrm{ij}}$ and $\mathrm{q}$, respectively. For a rectangle pulse of duration $\Delta \mathrm{t}$,

$Q(\omega)=\operatorname{sinc}\left(\frac{\Omega_{t} \omega}{2 \pi}\right)$ 
Where $\operatorname{sinc}(x) \equiv \frac{\sin (\pi x)}{\pi x}$. For a Gaussian pulse with temporal FWHM, $\Delta$ t, we have

$Q(\omega) \propto \exp \left(-\frac{\sqrt{2} \Delta_{t}^{2} \omega^{2}}{32 \ln 2}\right)$

The top row of the figure shows these $Q$ envelopes for three values of $\Delta t$ that represent pulses near the impulsive, steady state, and mixed limits along with the FID envelope $|\Gamma+i \omega|^{-1}$. The bottom row shows the frequency dependence of the intensity of the coherence,

$\int\left|P_{i j}\right|^{2} \sim \int\left|\frac{Q(\omega-\Omega)}{\Gamma+i \omega}\right|^{2} d \omega$.

The fringes in the line shape are important in the impulsive regime but are suppressed at longer pulse durations by the FID bandwidth. 